\title{
La mine comme site artistique: un projet de Land Art en province Sud, Nouvelle-Calédonie. Témoignage et réflexion esthétique
}

The minig site as an artistic spot : a Land Art project in the South Province, New Caledonia. An account and an aesthetic reflection

\section{Florence Giuliani}

\section{OpenEdition}

Journals

Édition électronique

URL : http://journals.openedition.org/jso/7145

DOI : $10.4000 /$ jso. 7145

ISSN : $1760-7256$

\section{Éditeur}

Société des océanistes

Édition imprimée

Date de publication : 15 décembre 2014

Pagination : 165-174

ISBN : 978-2-85430-118-2

ISSN : 0300-953x

\section{Référence électronique}

Florence Giuliani, « La mine comme site artistique: un projet de Land Art en province Sud, NouvelleCalédonie. Témoignage et réflexion esthétique », Journal de la Société des Océanistes [En ligne], 138-139 | 2014, mis en ligne le 15 décembre 2017, consulté le 02 mai 2019. URL : http:// journals.openedition.org/jso/7145; DOI : 10.4000/jso.7145 


\section{La mine comme site artistique: un projet de Land Art en province Sud, Nouvelle-Calédonie. Témoignage et réflexion esthétique}

par

Florence GIULIANI ${ }^{*}$

\section{RÉSUMÉ}

Dans le récit d'une recherche esthétique à partir de la commande d'une auvre de type Land Art par la province Sud de Nouvelle-Calédonie, pour un terrain de mine orpheline, Florence Giuliani, artiste plasticienne travaillant depuis une vingtaine d'années en Nouvelle-Calédonie, retrace les différentes étapes d'un projet qui a vu sa réalisation inaugurée le 20 décembre 2013, sur le site du mont Goumba sur la commune du Mont-Dore.

MoTS-CLÉs : mines, Land Art, histoire

Le récit de la démarche qui m’a permis d'aboutir aux différentes phases de conceptualisation artistique pour ce projet, au fur et à mesure qu'il se précisait, se fait ici en trois points, allant de la manière de penser la mine comme un site artistique à la réalisation du projet et à la constitution de l'équipe de travail, en passant par l'étape de modélisation du projet.

\section{Penser la mine comme site artistique}

Entre nature et impact de l'action humaine sur des sites naturels, des lieux comme les mines orphelines sont tout aussi chargés d'histoire que de caractéristiques géographiques très marquées.

\begin{abstract}
Sculptor and installation artist, Florence Giuliani - who has been working in New Caledonia for over 20 years - provides an account of an aesthetic investigation she undertook for a project commissioned by South Province, New Caledonia. The author discusses the different development stages of a Land Art project, launched on 20 December 2013, located on an abandoned mining site on Mount Goumba (Mount-Dore).
\end{abstract}

KeYwords: mines, Land Art, history

On parle de "mine orpheline" lorsqu'il est impossible d'identifier le propriétaire d'un site minier afin d'en reprendre l'exploitation. Le terme désigne autant un vide juridique qu'un abandon géographique; la revégétalisation de ces sites est quasi impossible sans intervention humaine (Sarrailh, 2002). Ce vide appelle des interrogations qui sont autant de tentatives de mises à jour de récits : Pourquoi a-t-elle été abandonnée? Qui était propriétaire de cette mine ? Qui y travaillait ? etc. Lieux d'exploitation minière ayant été abandonnés sans plus y revenir, les mines orphelines portent les stigmates, non seulement de l'exploitation minière elle-même, mais aussi de l'érosion qui s'en est suivie. Les travaux scientifiques décrivent le phénomène : 
"La destruction du couvert végétal des vastes superficies soumises aux exploitations forestières, minières et aux incendies qui leur sont liés, a pour conséquence une érosion très active [...]. Les particules arrachées transitent par les cours d'eau pour sédimenter par gravité depuis les estuaires jusqu'aux pentes externes du récif où parviennent les particules les plus fines [...]. Ce processus d'hypersédimentation est à l'origine de la destruction d'habitats marins. " (Richer de Forges et Pascal, 2008 : 95-112).

Les sites de mines orphelines en Nouvelle-Calédonie sont porteurs de cet impact majeur sur la biodiversité mais aussi d'une histoire humaine riche et paradoxale qui conjugue plusieurs types d'approche de l'environnement par différentes civilisations ayant des conceptions du lien de l'humain à la nature qui l'entoure radicalement différentes.

Pour les caractériser de façon sommaire, j'appellerais ces différentes approches: "logique d'identification " et "logique d'exploitation ». Ce sont ces deux notions, avec le choix de partir de l'idée de "civilisations» plus largement que de «cultures» qui m'ont permis d'orienter ma recherche esthétique. Pointer la différence entre " logique d'exploitation» et "logique d'identification " permet de souligner la disparité des considérations et priorités selon le groupe humain auquel on appartient, ici européen ou kanak. L'histoire minière en Nouvelle-Calédonie est marquée par des hommes comme Garnier, ingénieur découvreur d'une nouvelle sorte de nickel qui porte son nom, "la garniérite ", le premier à avoir déposé un brevet pour l'exploitation industrielle du nickel calédonien. Higginson, également une figure marquante, homme d'affaire peu scrupuleux qui, comme le souligne le sous-titre de l'ouvrage qui lui est consacré par Anne-Gabrielle Thompson, est un "spéculateuraventurier à l'assaut du Pacifique " (Thompson, 2000 : sous-titre).

Elle est également caractérisée par l'emploi de mains-d'œuvre d'origines très diverses, dont les descendants forment les différentes communautés ethniques du pays et dont les conditions de travail donnent sens également au choix de parler de "logique d'exploitation ", comme le souligne la description suivante :

"Pendant un siècle, des milliers d'ouvriers venus d'Asie, d'Europe et de Polynésie sont employés sur les mines. Les forçats, prêtés par l'administration pénitentiaire, puis les engagés volontaires d'Asie, permettent aux sociétés minières de se développer. La colonie comptera jusqu’à 14535 engagés asiatiques en 1923, c'est-à-dire trois mineurs sur quatre, qui vivent dans des conditions extrêmement pénibles." (Pitoiset, 2008 : 11)

Pour ce qui est du rapport singulier aux lieux des Kanak, je me suis rapprochée de M. Georges Mandaoué, homme politique et, surtout, an- cien membre du sénat coutumier (président 2001-2002), puis, en charge de l'animation des secteurs de l'identité kanak et des affaires coutumières au gouvernement de la Nouvelle-Calédonie (depuis mars 2011) :

«J'ai parlé de l'esprit de la terre parce que cette notion renvoie au plus profond de notre spiritualité. [...] Le rapport entre l'esprit et soi est toujours là, quel que soit l'endroit où l'on se trouve. Il va nous révéler des choses par rapport à cet endroit et ses connaissances vont se cumuler à celles que l'on possède déjà. Par exemple, si durant un siècle tes ancêtres ont vécu dans la chaîne, en montagne, toi, tu as appris à fonctionner avec les éléments reliés à cet environnement, pour te nourrir de la cueillette, la pêche dans les rivières, la chasse, te soigner avec les plantes médicinales qui t’ont été révélées... " (Mandaoué, 2009 : 18-19).

On saisit mieux ici l'écart entre deux conceptions de la terre. Or la gageure de la proposition qui m’était faite m’obligeait à penser possible l'intégration de ces points de vue apparemment irréconciliables. Un article de l'anthropologue Pierre-Yves Le Meur donne des éléments de réponse quant à une possibilité de sens renouvelé :

"Il s'agit plus exactement de concevoir la relation foncière sous deux angles symétriques : la terre comme ressource, potentiellement exploitable et appropriable ("la terre nous appartient"), la terre comme source de vie, principe de fertilité ("nous appartenons à la terre"). "(Le Meur, 2010 : 94).

Il s'appuie pour cela sur les travaux de Marylin Strathern sur la Papouasie Nouvelle-Guinée (Strathern, 2009) où la pression minière appelle à une urgente reformulation des termes de la propriété foncière :

"Marylin Strathern n'oppose pas ces deux points de vue sur la base de dichotomies simplistes (occidental/ non occidental, capitaliste/non capitaliste) mais propose de réfléchir en termes de créativité plutôt que de productivité : "Penser la terre comme créatrice et ses produits comme des créations". Le registre de la création, de la capacité créative est exactement celui que le champ juridique subsume sous la catégorie de droit de propriété intellectuelle. Il permet de penser la terre à la fois comme ressource matérielle et immatérielle. Le versant immatériel de la terre s'exprime par sa fertilité, son potentiel reproductif et créateur, tout autant que par un récit fait de cheminements, d'alliances, de savoirs et de savoir-faire constitutifs d'un réseau liant la terre et ses possessions/possesseurs que sont les personnes passées, présentes et futures. » (ibid. : 94-95)

Il n'est pas un jour en Nouvelle-Calédonie qui ne soit marqué, d'une façon ou d'une autre, par l'enjeu minier, et c'est aussi de cette expérience au quotidien que je suis partie pour avancer dans une élaboration plastique. Un fait particulièrement marquant pour quelqu'un qui, comme 
moi, habite à la périphérie de la ville de Nouméa : à l'entrée de la ville, la vieille usine de la SLN (Société Le Nickel) accueille, entre beauté paradoxale des sites industriels et lâchers de fumées toxiques et colorées, tous ceux qui s'aventurent dans la ville. Elle est à l'avant, en proue, comme pour dire l'importance de l'histoire minière avant toute autre histoire dans ce pays.

En m'interrogeant sur ce qui pouvait bien donner cette importance à cette activité-là précisément, en dehors du fait économique, j'en suis arrivée à la conclusion qu'une part d'inconscient lui était intensément liée.

Dans le lexique minier, c'est le terme "veine" qui s'est imposé à moi comme étant le plus approprié pour dire ce qui se joue ici d'essentiel.

Il m'a inspiré le texte d'intention du projet destiné à mes interlocuteurs de la province Sud.

En parallèle au cheminement qui a été le mien pour dégager une notion sur laquelle je pourrais élaborer une conceptualisation plastique dans le cadre particulier de l'intervention sur site de mine orpheline, je me suis familiarisée avec le travail de certains artistes du Land Art qui ont œuvré de façon monumentale - une des contraintes majeures du projet étant qu'il devait pouvoir se voir de loin.

Parmi les artistes du Land Art, ou possiblement associés à celui-ci, la démarche artistique de James Turrell et son commentaire par Georges Didi-Huberman m'attiraient, notamment, le propos sur l'artiste dans son lien à l'environnement et son geste dans celui-ci :

"L'artiste est inventeur de lieux. Il façonne, il donne chair à des espaces improbables, impossibles ou impensables: apories, fables topiques." (Didi-Huberman, 2001 : quatrième de couverture).

Mais c'est la figure de Michael Heizer qui s'est imposée à moi comme étant la source d'inspiration, en quelque sorte tutélaire, la plus probante.

Michael Heizer, artiste actuel majeur du Land Art, qui a souvent œuvré dans des lieux de nature isolés dont, parfois, il a inventé des archéologies, a retenu mon attention plus particulièrement puisque, partant de l'idée de l'évocation de succession de civilisations et de leur relation à la nature, mais aussi de l'intention de dire quelque chose de cela dans le monde d'aujourd'hui, il me fallait pouvoir conjuguer des formes qui évoquent archéologie et contemporanéité dans le même temps, ce qui est le propre du travail de cet artiste.

Son œuvre intitulée City, "ville-sculpture" comportant des volumes de types murailles d'environ 1 mile de long dans le Comté de Lincoln dans le désert du Nevada aux États-Unis, m'a semblé pouvoir me donner la base d'un choix esthétique.
Michael Heizer dit de son œuvre Double Négative, deuxième référence pour moi dans sa production, qu'elle :

«[...] est la description littérale de deux incisions, mais elle a des implications métaphysiques puisqu'un double négatif est impossible. Il n'y a rien et pourtant, c'est toujours une sculpture. » (Heizer, $1970: 4$ )

L'audace de cette démarche qui consiste à creuser dans un paysage me frappait : la part inversée de ce qui pourrait être monumental et visible de loin et devient monumental mais en creux et dont les traces visuelles accessibles (photos, vidéos...) laissent deviner un impact peut-être plus impressionnant encore par l'absence ou la disparition d'une forme qui a pu être gigantesque que celui de la forme "présente » elle-même.

J'estimais qu'en faisant un parallèle avec le travail de cet artiste, je pourrais inscrire la mine calédonienne dans une démarche de type "Land art» et je réussirais à avancer dans la bonne direction. City et Double negative (Brown, 1984) de Michael Heizer me donnaient des clefs pour proposer un agencement de volume en plein et en creux qui pouvaient correspondre à l'aspect sillon/veine de l'exploitation du minerai luimême et à l'aspect de construction monumentale laissé par les traces de cette exploitation.

\section{Modélisation du projet}

En effet, ma préoccupation majeure était qu'il me fallait trouver une expression plastique qui englobe plusieurs points essentiels du projet, tels qu'ils se présentent dans le premier dossier rendu à mes interlocuteurs de la province Sud, à l'intention de la présidente de la Province, en février 2013, ici résumé :

«Pré-projet d'installation in situ sur mine orpheline :

veines métalliques

veines des mineurs qui gonflent sous l'effort

veine comme passage des ancêtres dans un paysgrand corps

veine artistique

Que dit-on lorsque l'on parle de 'veine'

'avoir de la veine'

'trouver une veine'

'trouver la veine'?

Parler médical, infirmier, parler du chercheur de métal précieux, parler populaire...

D'une conception du monde comme grand corps traversé par des esprits qui le créent, l'animent, le visitent, à celle d'un monde dont les ressources naturelles sont exploitables, d'abord à merci et aujourd'hui avec, dans quelques cas, une conscience de la nature comme un grand corps, habité par une diversité biologique à préserver, à protéger. 
Qu'est-ce qui irrigue un projet, de pays, de modèle économique, de modèle écologique? L'histoire de la mine en Nouvelle-Calédonie est celle d'une destruction de paysage et d'une construction économique, des entrailles de la terre laissées béantes, épuisées, et d'un monde qui accède à la modernité. Une perte, un gain.

Aujourd'hui, à un moment charnière de son histoire, la Nouvelle-Calédonie continue de plus belle son histoire minière. Plus que jamais, dans ce contexte économique et politique, il est nécessaire de parler de l'humain et du paysage, d'une écologie et d'une poétique.

Trouver une nouvelle veine pour continuer l'histoire et, dans l'espoir d'un mieux-être collectif, qu'avoir de la veine ait du sens pour tous et chacun, y compris le paysage.

Une écologie pensée est porteuse d'une sève qui pourrait bien être celle d'une nouvelle veine qui nous aille, qui nous rassemble. [....]

Le projet artistique que la province Sud de la Nouvelle-Calédonie met en route aujourd'hui naît de la volonté que paysage et peuple de ce pays se rassemblent autour de l'idée de transformation/ métamorphose plutôt que de déchirure/ destruction.

Cela est possible, mais demande que soient mêlés, pour un baume sur les blessures, bien des ingrédients rares : paroles recueillies d'un vécu réel - ni magnifié, ni occulté - qui émerge et des blessures et de la construction dite pionnière de ce pays, de n'en écarter aucune et de s'inspirer des savoirs de toutes les communautés présentes en Nouvelle-Calédonie pour panser. C'est un début. Il nous faut certainement un mythe commun, tant il est vrai que les groupes humains fondent leur homogénéité d'abord ailleurs que dans la réalité matérielle, économique : il nous faut un projet qui ait sens pour tous. Un projet de civilisation plus encore qu'un projet culturel.

Dans l'histoire de l'art du Xx ${ }^{\mathrm{e}}$ siècle, le Land Art tient la place très particulière qu'on inventée, en précurseurs, l'art minimal, entre autres mouvements, et des personnalités comme Joseph Beuys, Alan Sonfist... Puis Robert Smithson, Christo et Jeanne-Claude, Richard Long, Andy Goldworthy [...]

Le choix d'un type d'intervention artistique inspirée du travail de Michael Heizer se fait en partant des constats, d'une part, de la grande richesse de l'histoire des mines et, d'autre part, des étonnantes structures visuelles laissées par l'exploitation des minerais.

Le paysage laissé par l'exploitation minière est d'emblée associé à une défiguration, une désolation... Or une intervention qui s'architecture en partant des caractéristiques d'un site tel qu'il est permet, par un biais, de lui donner une configuration nouvelle qui se met à évoquer tout autre chose.

On aborde, ici, l'histoire d'une construction. Celle d'un pays et celle d'un paysage. Notre époque permet un recul historique et demande une vision d'avenir. Cette intervention/construction qui reste ouverte doit pouvoir évoquer, dans le même temps, une archéologie et une contemporanéité.

Ici l'acte artistique posé ne clôt pas le sens. Il donne des indications :

- le sillon, la veine, la circulation qui est la richesse du pays, matérielle et immatérielle.

- la mise en valeur des terrasses, liée à la récurrence de ces formes dans l'histoire du pays avec l'architecture de la culture du taro puis celle de la mine. [...]
- le tressage et/ou le tissage qui vient protéger, dans certaines des propositions, le sillon creusé. Ici c'est une intervention à moyenne échelle, dont la réalisation pourrait être confiée à un groupe de femmes kanak en lien avec le site sélectionné. Elle entre dans la logique du lien intime de l'humain à son lieu. Lélément tressé aura une durée de vie éphémère. Il est aussi une référence au "panier de richesse" des grandes coutumes Kanak qui permet de connaître et de dire sa richesse puis de la partager, de la distribuer. » (Extraits du dossier remis à la présidente de la province Sud, février 2013)

Aujourd'hui, j'ajouterais, en confirmant que cette mouture du projet correspondait bien, pour moi, à l'identité des lieux et des enjeux de la mine orpheline, une description de ce que je nommerais l' " esprit de réparation " que j'associe au projet d'intervention pour lequel j'avais écrit ce dossier. Je le décrirais comme étant la volonté de stopper l'hémorragie due à l'érosion majeure des sites anciennement exploités qui semble indiquer, par analogie, qu'il existe bel et bien une conscience qu'il est nécessaire de stopper la destruction de l'esprit du pays. On peut l'espérer tout du moins. La mise à jour de certaines réalités historiques, concernant les différentes communautés présentes en Nouvelle-Calédonie, permet d'aller plus avant dans la compréhension et l'acceptation des unes par les autres. C'est à ce mouvement de reconnaissance identitaire, par les communautés elles-mêmes par rapport à leur propre histoire et entre les communautés que j'associe la réhabilitation de sites naturels défigurés avec « esprit de réparation ". J'ajouterais également une référence à Augustin Berque et à son étude sur l'art de dresser des pierres dans le jardin japonais :

"Quand on dresse des pierres, eh bien, souffle un vent-sentiment qui se joue de la frontière entre l'agissant et l'agi, le sujet et l'objet, le lieu et l'œuvre. Il y a trajection: franchissement des limites auxquelles se tient le dualisme. " (Berque, 1997 : 212)

Ce que j'avais voulu essayer de traduire en tout premier lieu dans cette proposition, c'est une résolution plastique de la dualité entre l'exploitation d'un lieu, donc sa destruction, et sa construction, dans le même mouvement. La juxtaposition de conceptions du lien de l'humain à son lieu, au départ radicalement différentes mais trouvant, par une traduction visuelle, une " possibilité » et une forme dynamique incluant le(s) passé(s) des lieux et tendue vers un avenir, était bien ma priorité.

Je prolongerais également l'idée de l'architecture en terrasses comme forme récurrente de l'architecture humaine en précisant que chaque individu, à quelque civilisation qu'il appartienne, peut y retrouver des références qui font sens pour lui et ce que l'origine de la forme soit liée à une cosmogonie, une spiritualité et/ou une nécessité matérielle. 

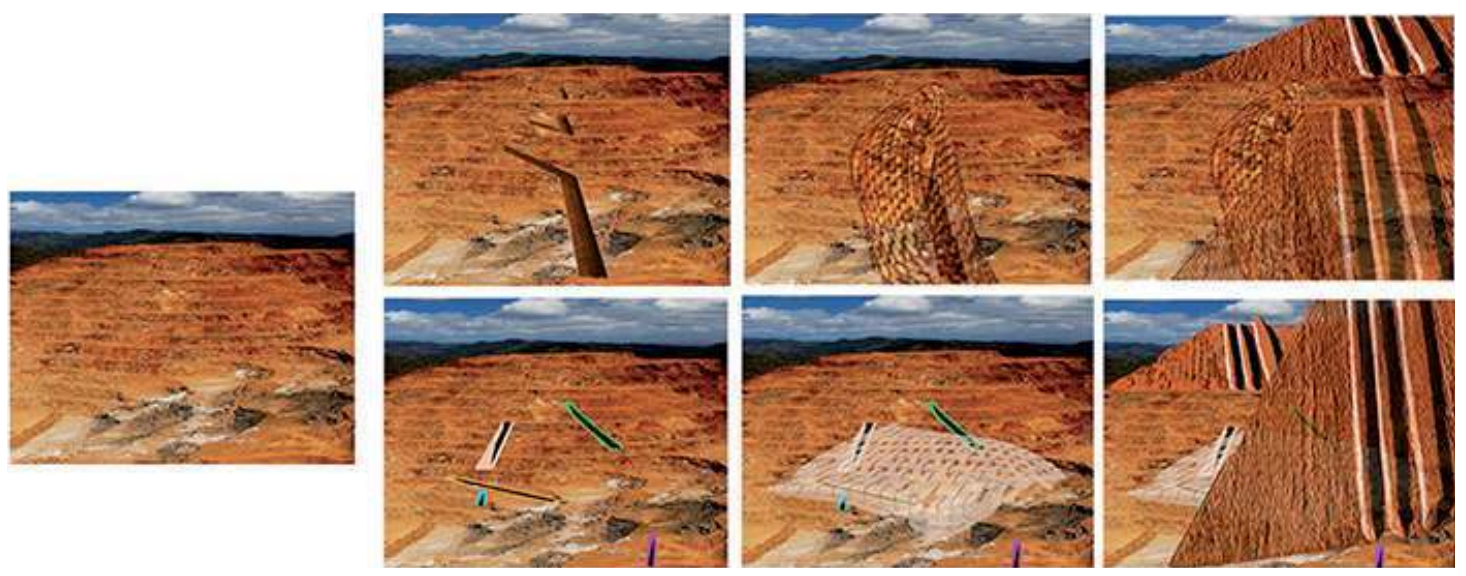

Figure 1. Essai visuel sur mine de Thio. Travail infographique comprenant des éléments visuels empruntés à Michael Heizer (cliché Giuliani, 2013, Nouméa, Nouvelle-Calédonie)

Un des défis du projet, sur un tel site minier en Nouvelle-Calédonie, est que le lieu est, d'une part, " chargé » spirituellement, comme tout lieu dans la conception kanak et ce, qu'il ait été habité ou pas, et, dans ce cas, le lieu a pu être un lieu de passage des esprits et/ou structuré par les chemins coutumiers ; il n'existe pas de lieu "où il n'y avait rien » en Nouvelle-Calédonie; d'autre part, transformé matériellement, avec des effets très importants et devenues dominants.

"Or ce territoire n'était pas vide. La Grande Terre et les Îles étaient habités par des hommes et des femmes qui ont été dénommés Kanak. Ils avaient développé une civilisation propre, avec ses traditions, ses langues, la coutume qui organisait le champ social et politique. Leur culture et leur imaginaire s'exprimaient dans diverses formes de création. Lidentité kanak était fondée sur un lien particulier à la terre. Chaque individu, chaque clan se définissait par un rapport spécifique à une vallée, une colline, la mer, une embouchure de rivière et gardait la mémoire de l'accueil d'autres familles. Les noms que la tradition donnait à chaque élément du paysage, les tabous marquants certains d'entre eux, les chemins coutumiers structuraient l'espace et les échanges... " (Signataires de la déclaration solennelle du 23 août 2002..., 2002 : 12-13)

Une fois ce dossier d'intention rendu à la présidence de la province Sud, faisant une large place à la structure en terrasses d'une part et au sillon et au tressage le recouvrant, d'autre part, une deuxième phase de recherche s'est effectuée qui m'a mise en contact avec de nombreux interlocuteurs, dans des domaines liés de très près à la réhabilitation des sites miniers.

Cette phase s'est déroulée une fois le choix du site d'intervention arrêté et l'intention clairement exprimée de procéder effectivement à la mise en place d'une ouvre ayant un aspect monumental visible de loin mais aussi un aspect plus intime allant dans le sens d'une réparation, d'un soin apporté à un lieu.
Le choix du site s'est arrêté sur celui de la mine des Barbouilleurs, sur la commune de Dumbéa, limitrophe de la ville de Nouméa, qui paraissait particulièrement indiqué pour la réalisation $\mathrm{du}$ projet pour plusieurs raisons : le site lui-même donnait à voir déjà, dans sa configuration, comme les restes archéologiques d'une cité et il était prévu de le réhabiliter dans l'année, ce qui permettait de conjuguer la mise en forme des volumes du projet et celui de la revégétalisation de la zone.

En effet l'aspect revégétalisation faisait aussi partie du projet et il était convenu que la réalisation inspirée du Land Art, par nature éphémère, surtout pour sa partie "intime", serait, d'une certaine façon, pérennisée par la végétation qui la recouvrirait. Ce choix d'une ouvre qui, à terme, disparaîtrait sous la végétation, était particulièrement important dans l'esprit de mes interlocuteurs. C'est précisément cette particularité qui semblait pouvoir décider les partenaires de la province Sud en matière de réhabilitation de sites miniers à accepter et suivre le projet.

\section{Réalisation du projet, constitution des équipes de travail}

C'est donc en partant de ce qui parait déjà évoquer les vestiges d'une ancienne cité sur ce site de la mine des Barbouilleurs que j'ai proposé une intervention en plusieurs points à la présidente de la province Sud en mars 2013. Cette description complétait le premier dossier rendu qui avait établi la conception générale du projet sur ce type de site et ses implications, je n'y revenais donc pas et me focalisais sur les nouvelles données dues aux caractéristiques du site précis.

C'est cette proposition pour le site de la mine des Barbouilleurs qui comportait les éléments les plus en lien avec l'histoire ouvrière liée à la mine 


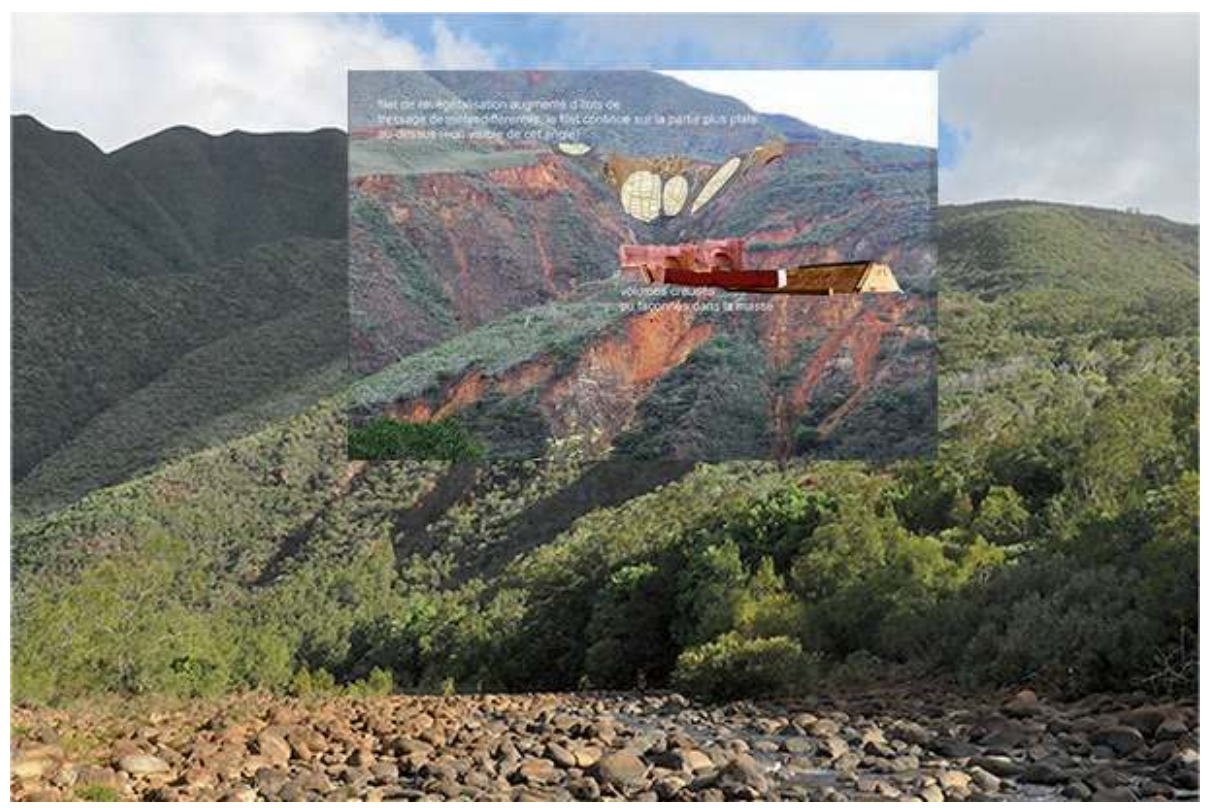

Figure 2. - Travail infographique comprenant des éléments visuels empruntés à Michael Heizer (cliché Giuliani, 2013, Nouméa, Nouvelle-Calédonie)

puisque j'envisageais de faire apparaître, par endroits, des éléments évoquant des baraquements de mineurs. Par contre, la dominante des terrasses avait disparu : le site, très encaissé n'en comportait pas, en tout cas pas qui soient visibles de loin. La " cité ancienne " venait là remplacer la référence archéologique aux terrasses et me semblait convenir également, surtout pour un lieu jouxtant aujourd'hui des quartiers résidentiels, comme c'est le cas dans la commune de Dumbéa à proximité de l'entrée des routes menant à la mine.

En rencontrant des techniciens de la DIMENC (Direction de l'Industrie, des Mines et de l'Énergie en Nouvelle-Calédonie) ainsi qu'un bureau d'étude indépendant préparant le projet de réhabilitation du site de la mine des Barbouilleurs, il a rapidement été évident que le choix du site avait été fait sans prendre suffisamment en compte l'extrême friabilité du terrain dans la zone, ni le fait que la réhabilitation d'un site minier nécessite que les volumes présents sur ce site soient aplanis de façon à contrôler l'impact des coulées d'eau, donc de l'érosion. Pour le projet artistique, il nous fallait rehausser les volumes ou, pour le moins, les confirmer, pour leur impact visuel à distance. Nous étions donc dans une sorte de contre-sens.

La rencontre, par la suite, d'un topographegéomètre qui avait fait le levé topographique de la zone confirmait l'impossibilité d'une opération du type prévu. Une alternative était proposée qui permettrait d'intervenir dans la dernière partie de la réhabilitation du site, en favorisant une mise en forme de type anamorphique. L'idée était de privilégier des points d'impact visuel qui donneraient une illusion de volume à partir d'un point particulier où seraient placés les visiteurs. Ce point pouvait être choisi au niveau d'un trou d'eau, situé sur la branche nord de la rivière Dumbéa, ayant une vue particulièrement nette sur la mine des Barbouilleurs.

Après rapport fait de ces nouvelles données à mes interlocuteurs de la province Sud, il a été finalement convenu de déplacer le lieu du projet : le projet de réhabilitation de la mine des Barbouilleurs, d'une complexité évidente en raison de la nature du terrain, pouvait tout à fait être reculé dans le temps et nuire, de ce fait aussi, à la réalisation du projet artistique.

Il a été décidé alors de déplacer le projet au mont Goumba, sur la commune du Mont-Dore, également limitrophe de la ville de Nouméa. Le mont Goumba offre l'intérêt majeur d'être situé juste derrière le complexe commercial le plus important de la commune et de permettre à un large public d'avoir un accès visuel facile à la zone d'intervention.

Par ailleurs, le mont est aménagé, depuis plusieurs années, par la commune du Mont-Dore, en lieu de randonnée et de découverte botanique. De multiples points de vue sur la zone sélectionnée pour l'intervention artistique sont possibles. Pour accompagner l'intervention, il est décidé d'organiser également une exposition mêlant œuvres d'artistes plasticiens inspirés par le mont Goumba, panneaux pédagogiques élaborés par une association spécialisée dans la revégétalisation, exemples de volumes de tressage créés par des tresseuses habitant la tribu de Saint-Louis, qui jouxte la zone du mont Goumba, ainsi que la création, par des lycéens de la commune, d'un 
volume inspiré par l'œuvre majeure du Land Art qu'est la Spiral Jetty de Robert Smithson.

Le mont Goumba, lieu facile d'accès et de présentation pour une œuvre dans la nature, a induit une inversion dans la proposition artistique en donnant la primauté aux éléments de tressage et en reléguant à un second plan les possibles volumes créés en rehaussant ou architecturant les masses de terre présentes sur le site. Cette proposition convenait à mes interlocuteurs, attachés à l'idée du pansement et très favorables au fait que ce serait des femmes de la tribu de Saint-Louis qui les fabriqueraient.

Gemma Ouaka, Marie-Christine Poitchili et Simone Moentéapo ont exprimé, chacune à leur tour, lors de mes visites sur place pour voir l'avancée de leur production, leur joie de pouvoir participer à un événement qui mette en valeur la tribu et qui leur permette de transmettre quelque chose de leur savoir, ne serait-ce que par la curiosité suscitée par les éléments inédits qu'elles fabriquaient. Cet événement leur permettait d'espérer que des jeunes filles de la tribu pourraient être amenées à essayer leurs techniques si le projet se poursuit en 2014, comme il en est question. Le fait qu'elles aient, pour cette occasion, mis au point des techniques nouvelles qui leur permettent de fabriquer des éléments très volumineux par rapport aux paniers et autres objets artisanaux qu'elles fabriquent habituellement, leur donne un élan pour l'avenir. C'est comme si, maintenant, il leur était possible de fabriquer de nouvelles formes qui correspondent mieux à ce à quoi les jeunes sont sensibilisés aujourd'hui. Ou, en tout cas, qui réussissent à susciter leur intérêt voire leur admiration malgré leur tendance à privilégier des expressions plus violentes. Ceci a d'autant plus d'importance que Saint-Louis est un lieu très particulier comme l'indiquent les témoignages qui suivent et que la tribu est régulièrement pointée comme ayant un fonctionnement problématique. L'histoire du lieu et sa géographie apportent bien des éléments d'explication à cet état de fait.

"Contrairement aux autres tribus, qui ont des racines millénaires, Saint-Louis fête tout juste ses cent cinquante ans. C'est en fait une création des Français. Pour loger les bagnards, qui, une fois libérés, se voyaient offrir des terres, l'administration coloniale a déplacé des populations kanakes un peu partout dans le pays et les a regroupées à Saint-Louis en 1859." (Tein, 2009)

La tribu est également particulière dans son emplacement géographique, sur la route principale reliant Nouméa au Mont-Dore :

" Si ça s'enflamme aussi vite ici, c'est aussi parce que beaucoup ont la rage. Pourtant, la vie n'est pas si mauvaise ici. Il y a la famille, il y a les champs. Mais le fossé est trop voyant entre nous et tout le fric qu'il y a Nouméa. [...] J'habite juste à côté, près du virage du pont de la Thy. Je regarde passer les Porsche Cayenne, les camions de minerai. Moi, c'est ça qui me fend le cœur. " (Témoignage de Yvéric, 23 ans, ouvrier agricole, 20/09/2009, http://www.humanite. fr/node/16676)

Gamma Ouaka, l'une des tresseuses du projet, a ainsi exprimé ce qu'impliquait le projet du mont Goumba pour elle et les jeunes autour d'elle:

"Les jeunes qui passent ici, ils sont étonnés de ce que l'on fait, ils n'en croient pas leurs yeux. Il y en a même qui s'approchent et nous posent des questions. J'ai ma petite-fille qui habite à côté et qui passe par ici souvent qui m’a dit: "Mamie, on dirait un soleil ce que tu fais, c'est beau, ça te ressemble". J'espère que je pourrai entrainer quelques jeunes à venir nous aider si on continue l'année prochaine. " (Gemma Ouaka, Saint-Louis,entretien 2013)

Cette nouvelle mouture du projet qui met en avant le tressage, pour sa partie réalisée en 2013, est présentée au public sur un panneau explicatif de la démarche dans l'exposition accompagnant l'intervention in situ. Ce panneau met l'accent sur « le lien, le tissu comme pansement sur une zone endommagée par l'activité humaine, par les humains, dans un ensemble d'éléments fabriqués avec patience et soin " (Giuliani, extrait du texte présentant l'installation).

Ainsi l'on retrouve bien, en partie du moins, l'esprit initial du projet, dans une mouture rendue plus simple et modeste par la transformation de celui-ci et l'inversion des priorités : éléments tressés placés dans une logique de pansement sur un site endommagé par l'action humaine avant revégétalisation puis, dans un second temps, volumes sculptés devenus les éléments à découvrir au cours d'une randonnée.

Le site du mont Goumba n'a pas été un lieu d'exploitation minière à grande échelle, il a été surtout un terrain de prospection. Dans une logique de sens qui est partie intrinsèque de toute élaboration artistique, cela résonne d'une façon particulière. Un lieu de prospection n'engage pas autant de paramètres humains signifiants que ne le fait un lieu d'exploitation même s'il connait aussi une histoire géologique et botanique très particulière et peut être très gravement endommagé comme le souligne l'extrait suivant d'une étude sur la biodiversité de Nouvelle-Calédonie :

«Les terrains miniers de Nouvelle-Calédonie occupent une superficie de $5500 \mathrm{~km}^{2}$, soit près du tiers de la superficie de l'île (Lafoy et al., 2003 ; Pelletier, 2007). Leur exploitation, qui s'est poursuivie sans interruption de la fin du XIX $x^{e}$ siècle à nos jours, débute par une prospection intensive destinée à localiser les gisements les plus riches et les plus accessibles. Cette prospection nécessite le percement de nombreuses pistes qui fragmentent l'écosystème et favorisent les départs de feu dans une végétation sèche, particuliè- 
rement sensible. » (Richer de Forges et Pascal, 2008 : 95-112)

Si l'impact environnemental est majeur, l'histoire humaine d'un site de prospection est moins vertigineuse que ne l'est celle d'un site d'exploitation. C'est sans doute ce qui a permis que l'intervention artistique se fasse de façon plus simple et concertée que ne l'aurait été une intervention sur un site de mine orpheline, forcément ancien lieu d'exploitation, comme il en était question au départ du projet, et cela, me semble-t-il, audelà des problématiques techniques.

En esprit, une intervention du type de celle prévue dans le premier dossier remis à la direction de la province Sud reste possible. Plus d'expérience du terrain minier, de la logique de la réhabilitation minière et de la revégétalisation, permettront, peut-être, à terme, de faire aboutir une intervention plus volumineuse et spectaculaire sur un site d'ancienne exploitation minière. Sans doute faut-il aussi que l'histoire minière du pays soit envisagée avec plus de facilité dans sa complexité et sa part de violence pour pouvoir réaliser un projet d'une envergure plus importante. Il me semble, en effet, qu'on ne peut produire une forme d'art spectaculaire, visible de loin, sans que l'histoire liée à cette forme ne soit clarifiée pour ses commanditaires comme pour l'artiste sollicité(e), et aussi pour le public qui sera témoin de cette forme.

Si l'aspect « civilisationnel » était intrinsèque au projet de plus grande envergure mettant en avant une archéologie, c'est plutôt d'aspect " culturel " qu'il est question ici, engrangeant moins de questionnement éthique et moins d'impact artistique en présentant, comme éléments principaux de la réalisation, des éléments de tressage, que l'on associe avec facilité aux pratiques artisanales et culturelles des femmes kanak.

La question de savoir ce que l'on a fait, dans la réalité de l'histoire du pays actée par les traces laissées dans son paysage, reste peu abordée ici, ou ne l'est "que " par le biais environnemental. Les réalités humaines plus violentes, et de l'exploitation des ressources d'un pays, de l'utilisation d'une main d'œuvre marquée par l'histoire de la colonisation, conjuguée aujourd'hui à celle de la mondialisation, et de l'histoire ouvrière, reste en marge.

Des écueils majeurs existent qui pourraient entraver la recherche nécessaire à la conceptualisation plus précise et à l'explication des choix d'une intervention plus monumentale dans le paysage minier calédonien : le manque d'informations et de témoignages directs de personnes ayant fait partie de la main d'œuvre d'origines multiples qui a travaillé sur les mines orphelines mais aussi le rapport à l'histoire et à la mémoire. Pour ce dernier, et dans le cas de l'histoire minière en Nouvelle-Calédonie, il me semble intéressant de juxtaposer les idées suivantes, en choisissant tout d'abord un propos général sur la relation au passé :

« [...] Paul Ricœur nous donne, avec La Mémoire, l'Histoire, l'Oubli, une somme philosophique pourtant d'une cohérence exemplaire, assise sur un processus méthodique d'examen de ce qui fonde la relation de l'homme au passé, présentée assez vite comme une relation incomplète et obscure ("l'énigme d'une représentation présente du passé absent", p. 511), marquée à la fois par la séparation de fait de l'homme d'avec son passé et par l'éclatement de la terminologie permettant de penser cette séparation. » (Vigier, 2000 : chap. 1)

À cela s'ajoute une question de méthode d'appréhension de l'histoire qui fait encore très certainement défaut dans le contexte calédonien :

«Se pose dès lors un autre problème, celui de l'épistémologie de l'histoire, par laquelle se trouve posée la question des rapports de la mémoire et de l'histoire comme "discipline scientifique et littéraire" (169), envisagée dans ses trois phases définies par Michel de Certeau: la phase documentaire (des témoins oculaires aux archives), la phase explicative/compréhensive (interprétation et recherche des causes) et la phase représentative (mise en forme littéraire ou scripturaire). " (ibid.)

Je me référerai ensuite au travail d'Eddy Banaré qui, à partir du récit minier calédonien, a particulièrement mis en évidence la nécessité d'un imaginaire fondateur héroïque pour une communauté, quand, par ailleurs, son histoire rend difficile qu'elle ait d'elle-même une image positive :

«Les mineurs deviennent une référence, s'imposent comme une nouvelle figure d'autorité. Ils pensent, émettent leur avis et sont en mesure d'annoncer et de garantir la prospérité. [...] Il n’y a pas vraiment eu de ruée vers l'or en Nouvelle-Calédonie comparable à l'Australie ou à la Californie. [...] Au lieu du rush c'est le doute qui semble progressivement s'installer [...] Le portrait du pionnier est né de là. L'Australie et la Californie ne sont plus enviées mais désormais citées en exemples de l'imprudence que les colons de la Nouvelle-Calédonie doivent éviter. C'est un appel à la sagesse. [...] Il s'agirait de dire à chaque colon qu'il est le personnage d'une grande chanson de geste, ou d'une épopée, qu'il participe à étendre le royaume. [...] Le portrait du prospecteur en martyr et presque messie est fondateur d'un imaginaire, c'est une vision romantique. Il y a, en Nouvelle-Calédonie, une refondation identitaire qui relie au pionnier plutôt qu'au bagnard »(Banaré, 2011, document audio)

On voit bien là toute la difficulté à mettre à jour suffisamment de faits avérés et à bousculer des mythes créés par la nécessité d'envisager sa place dans un espace de façon positive pour pouvoir concevoir un avenir et prospérer. Une forme monumentale dans cet espace amènerait peut- 


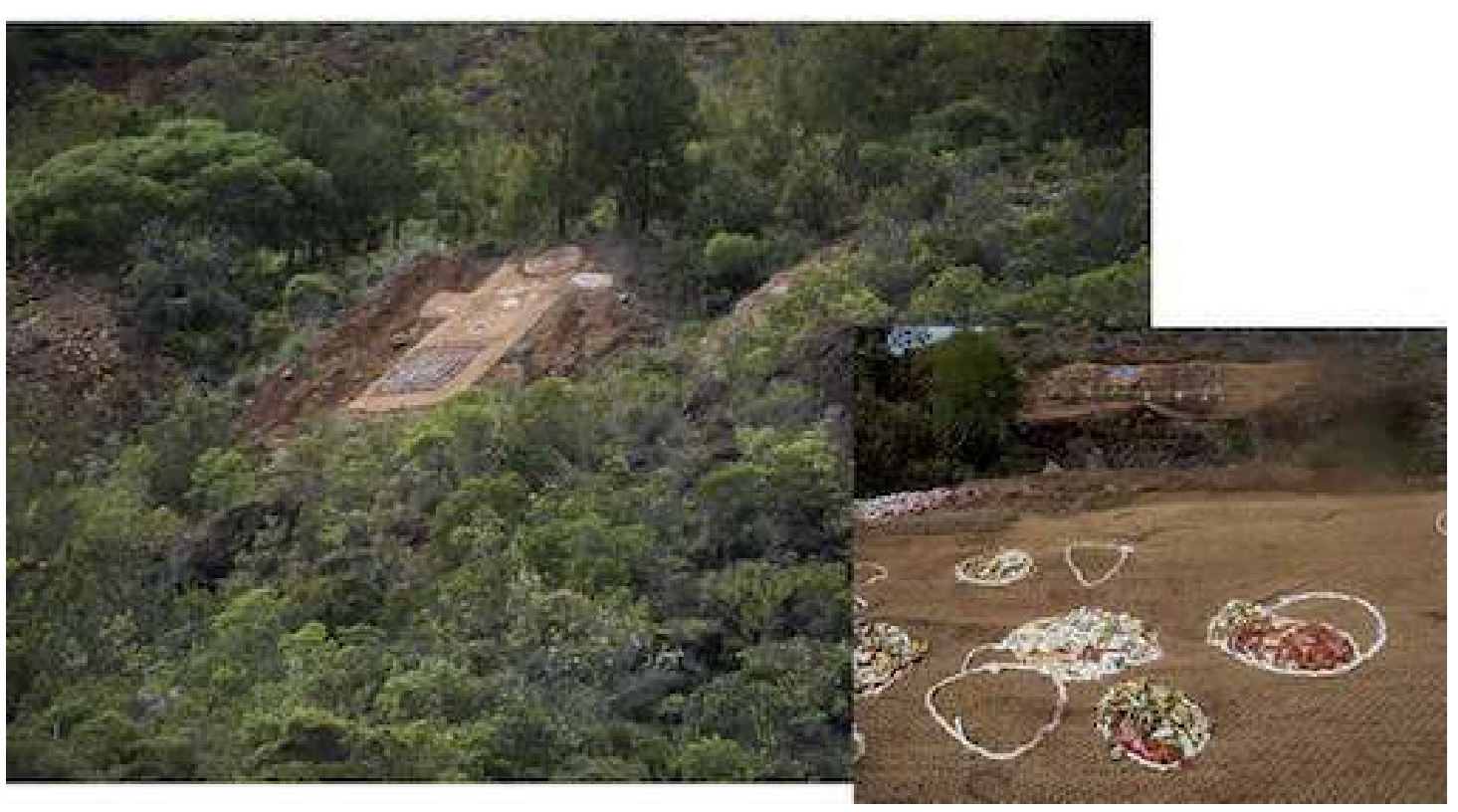

Figure 3. Intervention in situ sur le Mont Goumba(cliché JCR, 2013, Mont Goumba, Nouvelle-Calédonie)

être trop de questions à la surface d'un ensemble qui comporte, encore aujourd'hui, énormément de choses tues, ou racontées avec la nécessité d'un imaginaire héroïque.

Le projet d'intervention sur le Mont Goumba possède, une fois cela dit, un charme et un aspect accessible très émouvant. Il est une étape qui mêlent suffisamment de paramètres artistiques, associatifs, culturels, politiques et environnementaux pour être marquée du sceau de "l'esprit de réparation » nécessaire à notre époque et à ce pays.

\section{Conclusion}

L'intervention sur site sur le mont Goumba s'inscrit dans une continuité de ma recherche de plasticienne. Elle entretient des liens forts avec une série d'interventions que j'ai pu proposer depuis quelques années, qui cherchent à restituer le lien puissant entre l'humain et son environnement. Elle s'inscrit également, pour moi, dans une réflexion au long terme, nourrie par la pensée d'Édouard Glissant, qui cherche à rendre perceptible une "créolisation", selon le terme utilisé par l'auteur:

« La créolisation est la mise en contact de plusieurs cultures ou au moins de plusieurs éléments de culture distinctes, dans un endroit du monde, avec pour résultante une donnée nouvelle, totalement imprévisible par rapport à la somme ou à la simple synthèse de ces éléments. » (Glissant, 1997 : 37)

Si un certain nombre de réalisations préalables à l'invitation faite par la province Sud de Nou-
velle-Calédonie de donner forme à une intervention de type Land Art sur un site de mine orpheline m’a donné une base de réflexion pour une conceptualisation artistique, c'est l'idée d'aboutir une forme qui puisse conjuguer une allusion au passé et une dynamique vers l'avenir qui m’a guidée. Les questions de mémorialisation et de réparation me paraissent devoir être abordées dès qu'il s'agit d'envisager un possible devenir de sites miniers abandonnés comme on en trouve en très grand nombre en Nouvelle-Calédonie mais aussi dans de nombreux autres pays du Pacifique. L'intervention sur le mont Goumba peut être une première étape de collaboration interculturelle pour une recherche de formes artistiques sensibles qui mettent en évidence la possibilité d'un avenir renouvelé de ces sites.

\section{BIBLIOGRAPHIE}

Banaré Eddy, 2012. Aspects du grand récit minier en Nouvelle-Calédonie, Nouméa, Centre culturel Tjibaou, Document audio 56 mn (http://mediatheque.adck.nc/mediath/ son.cfm? urlson $=\mathrm{mms}$ : $/ / \mathrm{srv}-\mathrm{opac} / \mathrm{confe}-$ rence/12_0412_Banare.mp3\&notice=31407).

Berque Augustin, 1997. Dresser les pierres, ou le lieu de l'œuvre, Communications 64 , 64, pp. 211-219 (http:/www.persee.fr/web/ revues/home/prescript/article/comm_05888018_1997_num_64_1_1980). 
Brown Julia (ed.), 1984. Sculpture in reverse: Michael Heizer, Los Angeles, Museum of Contemporary Art.

Didi-Huberman Georges, 2001. L'homme qui marchait dans la couleur (James Turrell), Paris, Éditions de Minuit.

Giuliani Florence, 2013. Notes et documents de travail, archives personnelles.

Glissant Édouard, 1997. Traité du Tout-monde, Paris, Gallimard.

Heizer Michael, 2012 (12/08). Une œuvre in situ de l'américain Michael Heizer dans les Alpes suisses, Valais, communiqué de presse, Fondation 'Air \& Art' (http://www.air-art. $\mathrm{ch} /$ heizer/dossierpress-fr.pdf).

Le Meur Pierre-Yves, 2010. La terre en NouvelleCalédonie : pollution, appartenance et propriété intellectuelle, Multitudes 41, pp. 91-98.

MandaOuÉ Georges, 2009. La perception kanak de l'environnement, Mwa Vee 63, pp. 16-23.

Pitoiset Anne. 2008. Le nickel en NouvelleCalédonie, Maison de la Nouvelle- Calédonie.

Richer de Forges Bertrand et Michel Pascal, 2008. La Nouvelle-Calédonie, un "point chaud " de la biodiversité mondiale gravement menacé par l'exploitation minière, Journal de la Société des Océanistes 126-127: Environnement dans le Pacifique Sud (J. Tri- chet et I. Leblic éds), pp. 95-112 (http://jso. revues.org/4052).

SARrailh Jean-Michel, 2002. La revégétalisation des exploitations minières : l'exemple de la Nouvelle-Calédonie, Bois et forêts des tropiques 272/2, pp. 21-31.

Signataires DE LA DÉClaration SOlENNELLE DU 23 AOÛT 2002 DU PEUPLE AUTOCHTONE KANAK AFFIRMANT SON DROIT SUR L'ESPACE ET LE PATRIMOINE Naturel DE Kanaky, 2009. Mwa Vee 63 (https://fr.groups.yahoo. $\mathrm{com} / \mathrm{neo} / \mathrm{groups} /$ kanaky/conversations/topics/4369).

Strathern Marilyn, 2005. Land: Intangible our Tangible Property?, Chesters Thimothy (ed.), Land Rights: The Oxford Amnesty Lectures 2005, Oxford, Oxford University Press, pp. 13-38.

Tein Christian, 2009 (20/09). Nouvelle-Calédonie. Saint-Louis, l'enfant terrible, Paris, L'Humanité (http://www.humanite.fr/ node/16676).

Thompson Anne-Gabrielle, 2000. John Higginson. Un spéculateur-aventurier à l'assaut du Pacifique: Nouvelle-Calédonie, Nouvelles-Hébrides, Paris : l'Harmattan.

Vigier Yves, 2000. Une refondation de la mémoire (CR de Paul Ricœur, La Mémoire, l'Histoire, l'Oubli, Paris, Le Seuil, L'ordre philosophique) (http://www.fabula.org/revue/ cr/76.php). 